\title{
Visual Characterization of Paper Using Isomap and Local Binary Patterns
}

\author{
Markus Turtinen, Matti Pietikäinen and Olli Silvén \\ Machine Vision Group, Electrical and Information Engineering Department \\ P.O.Box 4500, FIN-90014 University of Oulu, Finland \\ \{dillian,mkp,olli\}@ee.oulu.fi
}

\begin{abstract}
In this paper, we study how multidimensional local binary pattern (LBP) texture feature data can be visually explored and analyzed. The goal is to determine how true paper properties can be characterized with local texture features from visible light images. We utilize isometric feature mapping (Isomap) for the LBP texture feature data and perform non-linear dimensionality reduction for the data. These $2 D$ projections are then visualized with original images to study data properties. Visualization is utilized in the manner of selecting texture models for unlabeled data and analyzing feature performance when building a training set for a classifier. The approach is experimented with simulated image data illustrating different paper properties and on-line transilluminated paper images taken from running paper web in the paper mill. The simulated image set is used to get quantitative figures of the performance while the analysis of real-world data is an example of semisupervised learning.
\end{abstract}

\section{Introduction}

Texture analysis provides a useful set of tools for realworld paper inspection. Even though the texture analysis research is rather old, there still exist several different definitions of texture itself [13] and only a few very succesful exploitations of texture methods have been made in paper industry.

The biggest problem of using texture information in paper characterization is that the produced paper has a very homogenous appearance and the features should be very discriminative to detect relevant texture variations. The extreme conditions of paper mills cause variations for image appearance and the features describing the papers should be invariant with respect to the monotonic gray-scale variations caused by illumination changes, for example. Operating environments are usually hard to fix completely and the camera position can change between installations. These issues set requirements that features should be able to handle different rotations and scales as well. Also computational issues are important in this kind of applications, for example, to achieve a reasonable classification time for acquired samples.

Paper mainly consists of pulp, chemicals and flocs. Flocs are wood fibre bunches that are visible when looking through the paper against the light source. In paper characterization the main goal is to determine the proper- ties of flocs, like their size and shape. They characterize indirectly important properties of the paper such as formation, strength and printability. Paper making procedure has a lot of random processes resulting the flocs to randomly distribute over the paper. This causes the appearance of transilluminated paper to look like a stochastic texture even though some properties, like the direction of production line, are visible from the floc distributions.

Texture has been applied satisfactorily to paper defect detection [6] and some attempts have also been proposed for paper formation analysis [5, 3]. In this paper, we aim to determine the general visual quality of paper based on its textural appearance. This is basically what paper procuders do subjectively after production when analyzing light through papers with human eye.

No texture features or descriptors are availabe that are the best in all kind of texture analysis problems. Traditionally the best texture features for a given application are found by a comparative study using supervision and prelabeled data like in [11]. The data is analyzed and tested with different features and algorithms to find, for example, the most discriminating features. In real-world applications the labeling is usually very problematic causing much difficulties in analysis. In addition, the computational burden of optimization and search algorithms in feature analysis might be far too high.

LBP texture features [10] detect microstructres, like edges, lines, curves and flat areas from textures and have performed very well in many real-world texture analysis applications including paper characterization [14]. In that study the self-organizing map [7] was used as an user interface for classifying textured paper samples providing a useful view into the data. In this paper we study how visualization of LBP texture data with Isomap [12] can be utilized in feature analysis, training data selection and labeling. The dimensionality of the feature data is reduced with Isomap to two dimensions which then is visualized utilizing both manifold structure and original texture images. Visualization makes it possible to analyze and compare the performance of the features semi-supervisely without using labeled data. Isomap makes the higher-to-lower dimensionality mapping differently compared to the SOM and preserves relational distances between samples more faithfully revealing manifold structures of the data. The approach presented in [14] is more suitable for on-line use and classification tasks, but here we construct a tool for the early stage visual analysis of paper's texture and quality. The tool can be used when training a texture classifier and analyzing features with unlabeled data. 


\section{Proposed Approach}

The local binary pattern (LBP) operator offers an efficive way to analyze textures [10]. It has a simple theory and it combines properties of structural and statistical texture analysis methods. LBP is invariant against monotonic grayscale variations and recently it has been extended to have rotation invariant and multiscale properties. These matters make LBP very attractive to be used in paper texture analysis. Here we utilize different versions of the LBP operator to model the texture of paper in order to characterize different paper properties. We use the original 8-bit and more recent multiresolution versions of the operator and also include experiments with its rotation invariant version.

Isomap [12] is nonlinear dimensionality reduction method built on classsical MDS [4]. It tries to preserve the intrinsic geometry of the data utilizing geodesic manifold distances between all pairs of data points. The algorithm builds a neighborhood graph using $k$ nearest neighbors or certain distance threshold $\epsilon$. The distances are calculated along the manifold instead of direct Euclidean distances and finally the classical MDS is run for this distance data. There is a guarantee of asymptotic convergence to the true structure of the data when running Isomap with 'sufficiently large' sample sets [2]. Isomap can also recover the true intrinsic dimensionality of the nonlinear manifolds utilizing residual variance of projections, and when representing the data with global coordinates it can provide a very useful way to analyze high-dimensional observations [12].

Multi-dimensional LBP features can be visualized as 1D histograms but this does not provide very clear representation of the data especially when the amount of the samples to be visualized increases. Thus more intelligent and user friendly methods for visualizing the feature data are needed. One solution is to reduce the dimensionality of the data with Isomap and study how the features can cluster the data.

In Fig. 1 a, 256-dimensional LBP texture data of six samples from two classes are projected with Isomap. We can notice that the class boundary between these two classes can be easily drawn on the 2D Isomap plane (near $\mathrm{x}=0$ ). User can visualize the projection with original texture images and determine how features can discriminate different classes. Also a separate clustering algorithm, like $\mathrm{k}$-means, can be run on projection coordinates to help visual analysis, especially when there is some pre-knowledge about the number of classes, for example. This kind of visual exploration of multidimensional texture data can be utilized in several texture analysis tasks, including feature analysis, training of a classifier and unsupervised learning.

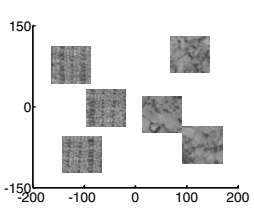

a)

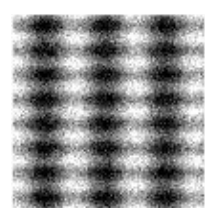

b)

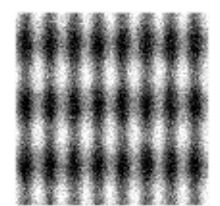

c)
Figure 1: 2d Isomap projection of six samples from two texture classes and examples of synthetized samples.
Visual analysis of the feature data is based on the assumption that similar data cluster close to each others in the feature space if the features used are discriminative enough. In dimensionality reduction this clustering information is mostly preserved when the manifold is mapped to 2-dimensions. Of course, the clusters might be more overlapped and divided into several parts in the projection. The main problem is to determine how badly the classes are mixed with each other. This can be done by visualization, also with unlabeled data.

The measure of residual variance can be used to quantify how well the low-dimensional Euclidean embedding captures the geodesic distances estimated from the neighborhood graph. It can be used to determine the optimal neighborhood size of Isomap algorithm [1] and to estimate the intrinsic dimensionality of the original data [12].

The true intrinsic dimensionality of the LBP texture data is usually much smaller than the original dimensionality, typically between three and fifteen. This supports the idea of using dimensionality reduction and visualization in texture data analysis. Kouropteva et al. [8] proposed a data visualization framework in which they first estimated the intrinsic dimensionality of the data and then reduced the dimensionality of the original data into that. After that they made pairwise plots of each new dimension and calculated the joint mutual information (JMI) criteria [9] from each plot to find the optimal visualization plane. Here we apply this kind approach to LBP feature data using Isomap for dimensionality reduction and estimation of the intrinsic dimensionality.

If the features tend to cluster samples into separate clusters, Isomap will also find separate manifolds from the data, especially with small neighborhoods (small $k$ or $\epsilon$ ). In these cases, the algorithm can be run separately on the disconnected manifolds. One can easily estimate the feature performance by visualizing the projections of submanifolds. Typically paper texture data is so homogenous that there will be no disconnected components in the low-dimensional embedding. The residual variance and JMI criteria can be utilized when finding the optimal mapping from the feature space to the $2 \mathrm{D}$. The goal is to select such a projection that provides useful visualization information and represents the original data optimally.

\section{Experiments}

In the experiments both simulated and real-world paper images were used. The appearance of paper was simulated with a simple periodic function $z(x, y)=r * \sin \left(t_{x} * x\right)+$ $\cos \left(t_{y} * y\right)$, where $\mathrm{r} \in[1,3], t_{x}, t_{y} \in[1,6]$ and $\mathrm{x}, \mathrm{y} \in[1,10 \pi]$. The dark areas of the synthetized image can be interpreted as flocs and the bright areas as voids. The images were categorized into 11 classes based on the "floc size" (so that $\left.t_{x}+t_{y} \in[2,12]\right)$. Totally 720 images were generated and Gaussian noise with $\mu=0.0$ and $\sigma=0.01$ was added to the images for creating more realistic $2 \mathrm{~d}$ paper textures. In the resulting images the form and in some cases the orientation of the "flocs" varied but the mean size of the "flocs" remained relatively constant. Fig. $1 \mathrm{~b}$ and c show examples 
of generated samples $\left(t_{x}+t_{y}=7\right)$

The real world data set consisted of paper images acquired from a Stora Enso papermill in Oulu, Finland. The running paper web was imaged for one and half month in December 2003 and January 2004 with 15 minute intervals between images using a fast high quality CCD camera. Images taken under downtime of the mill were rejected. The total number of samples was 2316 .

\section{Experiment \#1: visual feature analysis}

The synthetized data set was used in this experiment. A leave-one-out 3-NN classification with eight different LBP operators, $L B P_{8,1}, L B P_{8,1}^{r i}, L B P_{8,1}^{u 2}, L B P_{8,1}^{r i u 2}$, $L B P_{8,1+16,2+24,3}^{u 2}, L B P_{8,1+16,2+24,3}^{r i u 2}, L B P_{8,1+16,3+24,5}^{u 2}$ and $L B P_{8,1+16,3+24,5}^{\text {riu2 }}$ was made obtaining classification rates of $87.9,72.4,85.8,74.2,98.1,99.3,99.6$ and 99.9 percent, respectively. These results represent the ground truth for different features. Our aim is to demonstrate how visualization of the features with Isomap can be used when analysing the performance of different LBP operators.

For each feature set, its own Isomap projection was created using ten different neighborhood sizes $(k=[3,5, \ldots, 21])$. If the features cluster the data into separate clusters, Isomap can detect these efficiently when using small neighborhoods. With $k=3$, the number of connected components varied for 3-21 with different features. With larger neighborhoods $(k \geq 13)$ only one connected component was found with every feature set. The intrinsic dimensionality of the data was estimated from the residual variance versus Isomap dimensionality plots by searching for the "elbow" from the plot [12]. Then the JMI criteria was used to select the most informative dimensionality components when constructing the $2 \mathrm{D}$ projection.

Analysis of the residual variance plots suggests, for example, that with $L B P_{8,1}^{u 2}$ features the neighborhood size $k \geq 13$ should be used. With $L B P_{8,1+16,3+24,5}^{r i u 2}$ features $k=3$ provides the best result. Applying Isomap with $k=3$ for these data sets it found 5 sub-manifolds from $L B P_{8,1}^{u 2}$ (original dimensionality $=59$ ) and 19 sub-manifolds from $L B P_{8,1+16,3+24,5}^{\text {riu2 }}$ (original dimensionality $=54$ ) feature data. The largest sub-manifolds are shown in Figs. 2 a and $b$. The multiresolution and rotation invariant operator can discriminate classes (only 2 involved in this projection) very well, but with the $L B P_{8,1}^{u 2}$ operator there are confusions between classes ( 9 classes in the projection). In fact, sub-manifolds of the multiresolution operator could discriminate the classes nicely but some of the classes are fragmented into several sub-manifolds (19 clusters and 11 classes). Visualization of projections using the original textured images reveals rapidly that with $L B P_{8,1}^{u 2}$ features the classes are badly mixed. Figs. $2 \mathrm{c}$ and $\mathrm{d}$ show the projections using a larger neighborhood $(k=15)$. Now the local properties of the data are not preserved as well as with smaller neighborhoods and there is not that significant difference between projections. Both projections represent the classes interestingly constucting threads on the projection plane and with $L B P_{8,1}^{u 2}$ features the projection looks better than with the smaller $k$ value.

The results indicate that a proper visualization is useful when studying the performance of the texture features. The most informative projection for each feature set is found automatically, but in practice one should also visualize the projection obtained with a small neighborhood. This is because with small neighborhoods the Isomap may detect different classes automatically and represent the data using disconnected manifolds. This helps the visualization task because there are not so much data involved.

\section{Experiment \#2: creation of training set}

In the second experiment real-world image data was used. Our main aim was to develop a method used to train a classifier for classifying on-line paper samples to different 'quality classes' according to their appearance. The same features as in previous experiment were extracted and Isomap projections with the same parameters were constructed. With all features and sizes of neighborhoods the Isomap found only one connected component from the data. So the data does not have so clear class structures as in the previous experiments and textures are more homogenous and uniformly distributed.

For such a homogenous and hardly visualizable texture material as paper it is difficult to find the best LBP operator to be used. But when visualizing the projections carefully we notice that the roughness of the paper texture changes relatively smoothly when moving from manifold's one end to the other. The roughness correlates with the formation and printing properties of paper. Paper producers want their paper to be as uniform and smooth as possible to assure good quality and press properties of the paper.

By selecting a group of samples from different parts of the manifold for visualization we can try to locate the apparent 'class boundaries'. Fig. 3 shows the projection of $L B P_{8,1+16,2+24,3}^{r i u 2}$ labeled to five roughness classes. Because the visual labeling is quite subjective for such a material we first applied the k-means clustering to the projection points and then visualized and adjusted the boundaries. The left end of the projection represents smooth paper while the texture is more rough on the right end. After labeling we can train a classifier, like $k$-NN, using recently labeled samples as the training data and classify on-line paper samples with it.

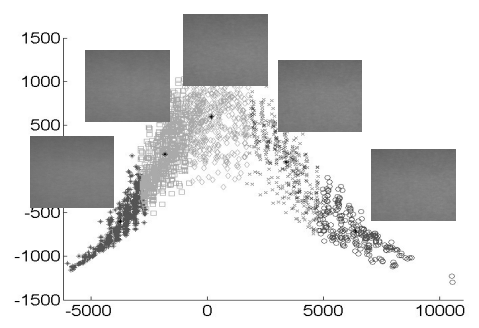

Figure 3: Isomap projection labeled to five roughness classes.

Visual labeling of the data and creation of training set can be utilized in various texture analysis tasks. The class boundaries can be found by visualizing the $2 \mathrm{D}$ projection (depending on features) and with effective user interface a 


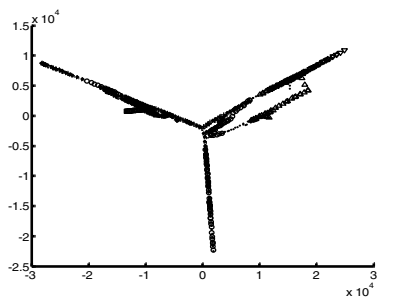

a)

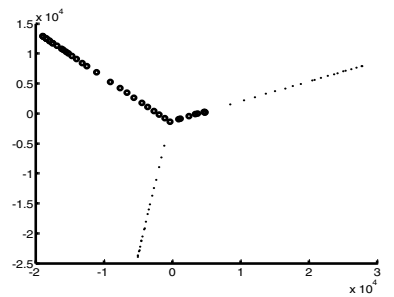

b)

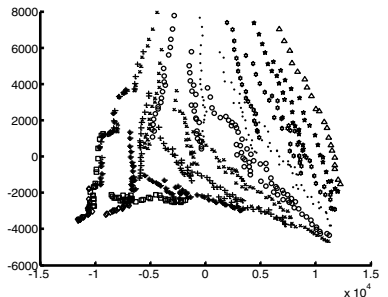

c)

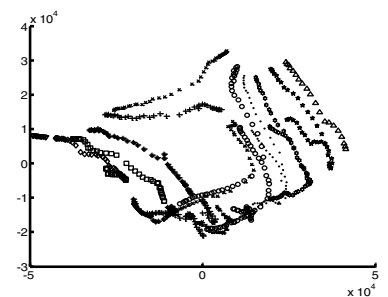

d)

Figure 2: Isomap projections of $L B P_{8,1}^{u 2}(\mathrm{a}, \mathrm{c})$ and $L B P_{8,1+16,3+24,5}^{r i u 2}(\mathrm{~b}, \mathrm{~d})$ features using $k=3$ (a,b) and $k=15$ (c,d) nearest neighbors.

large number of samples can be easily labeled. For human it is very difficult to see differences between homogenous samples. The visualization of Isomap projections is therefore useful with such a data because user can study data points locally and globally and determine if there is some change in texture apperances when moving on the manifold.

\section{Discussion}

Early data processing, including feature performance analysis, data labeling and training set creation for classification are laborious but fundamental tasks when characterizing textures in real-world environment. In this paper, we studied combined use of LBP texture features and Isomap dimensionality reduction method for analyzing transilluminated paper textures. The 2D Isomap projections of highdimensional feature data were visualized and utilized in the above mentioned tasks. Visualization of the 2D manifolds and original texture images reveals interesting information about the feature data and shows how well features can discriminate different texture samples. Visualization can be used when constructing the training set for classification and the user can select representative training samples and label them.

The approach was experimented with both synthetized and real-world paper texture images. With synthetized data it was shown that the visualization based method works well in feature performance analysis of different LBP operators. Real-world data was used to demonstrate capabilities in semisupervised learning: paper texture samples were visually labeled according to their appearance.

The analysis tool described in this paper can be utilized in various texture analysis problems and might provide very useful information about the inspected data. Paper textures are very homogenous, but it is a difficult texture analysis problem to characterize them. With the tool presented here, papermakers can perform visual grading for the produced paper more objectively and analyze what would be the properties of the produced paper. It is also possible to analyze separate off-line data sets and create a classification training set for characterizing papers according their textural appearance on-line.

Acknowledgments Financial support provided by the Infotech Oulu Graduate Schools is gratefully acknowledged.

\section{References}

[1] M. Balasubramanian, E. Schwartz, J. Tenenbaum, V. de Silva, and J. Langford. The isomap algorithm and topological stability. Science, 295:7a, 2002. Technical Comments.

[2] M. Bernstein, V. de Silva, J. Langford, and J. Tenenbaum. Graph approximations to geodesics on embedded manifolds. Technical report, Department of Psychology, Stanford University, USA, 2000. Available at http://isomap.stanford.edu/BdSLT.pdf.

[3] M. Bouyndain, J. Colom, R. Navarro, and J. Pladellorens. Determination of paper formation by fourier analysis of light transmission images. Appita Journal, 54(2):103-105, 115, 2001.

[4] T. Cox and M. Cox. Multidimensional Scaling. Chapman and Hall, London, 1994.

[5] T. Cresson and P. Luner. Characterization of paper formation, part 2: The texture analysis of paper formation. Tappi Journal, 73(12):175-184, 1990.

[6] J. Iivarinen and J. Rauhamaa. Surface inspection of web materials using the self-organizing map. In Intelligent Robots and Computer Vision XVII: Algorithms, Techniques, and Active Vision, pages 96-103, 1998.

[7] T. Kohonen. Self-organizing Maps. Springer-Verlag, Berlin, Germany, 1997.

[8] O. Kouropteva, O. Okun, and M. Pietikäinen. Data visualization with multiple machine learning methods. In Fourth IASTED International Conference on Visualization, Imaging, and Image Processing, pages 190-196, 2004.

[9] R. Moddemeijer. On estimation of entropy and mutual information of continuous distributions. Signal Processing, 16(3):233-246, 1989.

[10] T. Ojala, M. Pietikäinen, and T. Mäenpää. Multiresolution gray-scale and rotation invariant texture classification with local binary patterns. IEEE Trans. PAMI, 24(7):971-987, 2002.

[11] T. Randen and J. Husoy. Filtering for texture classification: A comparative study. IEEE Trans. PAMI, 21(4):291-310, February 1999.

[12] J. Tenenbaum, V. de Silva, and J. Langford. A global geometric framework for nonlinear dimensionality reduction. Science, 290:2319-2323, December 2000.

[13] M. Tuceryan and A. Jain. Texture analysis. In C. Chen and L. Pau and P. Wang (eds.) Handbook of Pattern Recognition and Vision, pages 207-248. World Scientific, Signapore, 2nd Edition, 1999.

[14] M. Turtinen, M. Pietikäinen, O. Silvén, T. Mäenpää, and M. Niskanen. Paper characterization by texture using visualization-based training. International Journal of Advanced Manufacturing Technology, 22(10-11):890-898, 2003. 